\title{
Increased Expression of Foxj1 after Traumatic Brain Injury
}

\author{
Gang Cui $\cdot$ Zhihua Yu $\cdot$ Zhen Li $\cdot$ Wei Wang $\cdot$ Ting Lu • \\ Chunhui Qian • Jiliang Li • Yunlin Ding
}

Received: 25 January 2011 / Accepted: 10 February 2011 / Published online: 24 February 2011

(C) The Author(s) 2011. This article is published with open access at Springerlink.com

\begin{abstract}
Foxj1 is a member of the Forkhead/winged-helix (Fox) family of transcription factors, which is required for postnatal differentiation of ependymal cells and a subset of astrocytes in the subventricular zone. The subpopulation of astrocytes has the ability of self-renew and neurogenic potential differentiated into astrocytes, oligodendrocytes, and neurons. However, its expression and function in the central nervous system lesion are not well understood. In this study, we performed a traumatic brain injury (TBI) model in adult rats and investigated the changed expression of Foxj1 in the brain cortex. Western blot and immunohistochemistry analysis showed that the expression of Foxj1 gradually increased, reached a peak at day 3 after TBI, and declined during the following days. Double immunofluorescence staining revealed that Foxj1 was co-expressed with MAP-2 and GFAP. In addition, we detected that Ki67 had the co-localization with NeuN, GFAP, and Foxj1. All our findings suggested that Foxj1 may be involved in the pathophysiology of brain after TBI.
\end{abstract}

Keywords Foxj1 - Traumatic brain injury · Rats . Neurogenesis

Gang Cui and Zhihua Yu contributed equally to this work.

G. Cui $(\bowtie) \cdot$ Z. Yu $\cdot$ Z. Li $\cdot$ W. Wang $\cdot$ T. Lu $\cdot$ C. Qian Department of Neurosurgery,

The First Affiliated Hospital of Soochow University, Suzhou 215006, People's Republic of China

e-mail: cuigang33@yahoo.com.cn

J. Li $\cdot$ Y. Ding

Department of Burn Surgery,

The First Affiliated Hospital of Soochow University,

Suzhou 215006, People's Republic of China

\section{Introduction}

In modern times, traumatic brain injury (TBI) contributes to a major cause of morbidity and mortality all over the world, especially for children and young adults (Langlois et al. 2006; Plesnila et al. 2007). More and more people have been paying attention to the pathophysiology of the trauma; however, to date, the mechanism of the inner progress is not yet fully understood and the effect of the therapeutics remains unsatisfied (Roberts et al. 1998; McKee et al. 2005). Previous experiment studies in vivo and in vitro have demonstrated that the event induced by TBI triggers not only the primary injury which occurs immediately after the damage, but also the second injury which develops for a long term proximately several days or weeks and plays an essential role in morbidity or mortality (Nortje and Menon 2004; Walker et al. 2009; Zweckberger et al. 2006). All the steps involved in the whole process will cause neuronal apoptosis, inflammatory reaction, and reactive astrogliosis, which lead to consequently tissue loss, impaired regeneration, and functional disabilities (Di Giovanni and Movsesyan 2005; McGraw et al. 2001; Raghupathi 2004). To investigate the pathological mechanism and the cellular and the molecular alteration after TBI appears important for the value in order to improve the outcome in clinical treatment.

Foxj1 is a member of the Forkhead/winged-helix (Fox) family of transcription factors, which has a conserved 100 amino acid DNA binding domain and plays important roles in cilia formation of the respiratory, reproductive, and central nervous systems (Clevidence et al. 1993; Hackett et al. 1995; Brody et al. 2000). Abnormal expression or targeted mutation of Foxj1 will result in an absence of cilia in the tissues and a defect in 
left-right axis determination of organs (Brody et al. 2000; Chen et al. 1998). Foxj1 suppresses T cell activity and thus spontaneous autoimmunity, through the repression of NF- $\kappa B$ activity (Srivatsan and Peng 2005). Foxj1 also inhibits the humoral immune response in B cells; FOXJ1 deficiency in B cells results in spontaneous and accentuated germinal center formation, implicated in the development of pathogenic autoantibodies and accentuated responses to immunizations (Lin et al. 2005).

Recent studies have shown that FoxJ1 is required for postnatal differentiation of ependymal cells and a subset of astrocytes in the subventricular zone (SVZ) and the subpopulation of astrocytes has the ability of self-renew and neurogenic potential differentiated into astrocytes, oligodendrocytes and neurons (Jacquet and SalinasMondragon 2009). We hypothesize that Foxj1 may be involved in the pathophysiological and biochemical progression after TBI, which is associated with the outcome of brain function and neurogenesis induced by injury.

In our study, we investigated the expression and the distribution of Foxj1 in the rat brain after injury for the first time. Our experiment is conducted to gain a brighter insight into the physiologic functions of Foxj1 in the normal brain and the cellular and molecular mechanisms underlying central nerve lesion and repair.

\section{Materials and Methods}

Animals and Surgery

Male Sprague-Dawley rats (weighing 220-275 g) were used in this experiment. After deeply anesthetized with chloral hydrate ( $10 \%$ solution), the heads of the rats were fixed in the stereotactic frame and a microknife was inserted into the right cortex under the aseptic condition $3 \mathrm{~mm}$ lateral parallel from the midline with an antero-posterior surgical incision (5 $\mathrm{mm}$ long, $3 \mathrm{~mm}$ deep, and $1 \mathrm{~mm}$ wide); thereafter, the scalps were sutured. Shamcontrolled rats were subjected the identical procedures to experimental rats except for being inserted with the microknife into the brain. After all the procedures, animals were returned to their cages and allowed freely to get food and water. Animals were housed under a $12 \mathrm{~h}$ light/dark cycle and the room temperature (RT) was kept at $37 \pm 0.5$. Experimental animals $(n=21)$ were killed at $12 \mathrm{~h}, 1,3,5$, 7,14 , and 28 days after injury. Normal rats $(n=3)$ and sham-controlled rats $(n=3)$ were sacrificed at 3 days. All surgical and animal care procedures were carried out in accordance with the Guide for Care and Use of Laboratory Animals (National Research Council, 1996, USA) and were approved by the Chinese National Committee to Use of
Experimental Animals for Medical Purposes, Jiangsu Branch.

Western Blot

After given an overdose of chloral hydrate, rats were killed at different time points post-operatively, and the cortex tissue surrounding the wound (extending $3 \mathrm{~mm}$ to the incision) as well as an equal part of the normal and shamcontrolled cortex were dissected out and stored at $-80^{\circ} \mathrm{C}$ until use. In order to prepare lysates, frozen cortex tissue samples were weighed and minced with eye scissors in ice. Then the samples were homogenized in lysis buffer $(1 \%$ sodium dodecyl sulfate (SDS), $1 \%$ Triton X-100, $50 \mathrm{mmol} /$ L Tris, $1 \%$ NP-40, pH 7.5, 5 mmol/L EDTA, 1\% sodium deoxycholate, $1 \mu \mathrm{g} / \mathrm{ml}$ leupeptin, $10 \mu \mathrm{g} / \mathrm{ml}$ aprotinin, and $1 \mathrm{mmol} / \mathrm{L}$ PMSF) and centrifuged at $12,000 \mathrm{rpm}$ and $4{ }^{\circ} \mathrm{C}$ for $20 \mathrm{~min}$ to collect the supernatant. After determined protein concentration with the Bradford assay (Bio-Rad), protein samples were subjected to SDS-polyacrylamide gel electrophoresis and transferred to a polyvinylidine diflouride filter membrane. The membrane was blocked with $5 \%$ milk without fat for $2 \mathrm{~h}$ and incubated with primary antibody against Foxj1 (anti-mouse, 1:1,000; Santa Cruz) or GAPDH (anti-rabbit, 1:1,000, Santa Cruz) at $4^{\circ} \mathrm{C}$ overnight. At last, the membrane was incubated with second antibody goat-anti-mouse or goat-anti-rabbit conjugated horseradish peroxidase (1:2,000, Southern-Biotech) for $2 \mathrm{~h}$ and visualized using an enhanced chemiluminescence system (Pierce Company, USA).

\section{Sections and Double Immunofluorescent Staining}

After defined survival times, rats were terminally anesthetized and perfused through the ascending aorta with $500 \mathrm{ml}$ of $0.9 \%$ saline, followed by $4 \%$ paraformaldehyde. After that, the brains were removed and postfixed in the same fixative for $3 \mathrm{~h}$ and then replaced with $20 \%$ sucrose for 2 3 days, following 30\% sucrose for 2-3 days. After treatment with sucrose solutions, the tissues were embedded in O.T.C. compound. Then, $10-\mu \mathrm{m}$ frozen crosssections were prepared and examined. All sections were first blocked with 10\% normal serum-blocking solution species the same as the secondary antibody, containing 3\% $(w / v)$ bovine serum albumin (BSA), and $0.1 \%$ Triton X-100 and $0.05 \%$ Tween- $202 \mathrm{~h}$ at RT in order to avoid unspecific staining. Then, the sections were incubated with both rabbit polyclonal primary antibodies for anti-MAP-2 (a marker of neurons, 1:1,000; Santa Cruz), anti-GFAP (a marker of astrocytes, 1:200; Sigma), and anti-Ki67 (a marker of cell division, 1:300; Santa Cruz), and mouse monoclonal primary antibodies for anti-Foxj1 (1:100; Santa Cruz), anti-NeuN (a marker of neurons, 1:500; Chemicon), anti- 
GFAP (a marker of astrocytes, 1:200; Sigma). Briefly, sections were incubated with both primary antibodies overnight at $4^{\circ} \mathrm{C}$, followed by a mixture of FITC- and TRITC-conjugated secondary antibodies for $2 \mathrm{~h}$ at $4^{\circ} \mathrm{C}$. The stained sections were examined with a Leica fluorescence microscope (Germany).

\section{Immunohistochemistry}

We blocked the sections with confining liquid consisting of $10 \%$ goat serum, $1 \%$ BSA, $0.3 \%$ Triton X-100, and $0.15 \%$ Tween-20 for $2 \mathrm{~h}$ at room temperature, then incubated with anti-Foxj1 antibody (anti-mouse, 1:100, Santa Cruz) overnight at $4{ }^{\circ} \mathrm{C}$. After incubation with the primary and the second reagents as the second antibody for 20 and $30 \mathrm{~min}$, respectively, at $37^{\circ} \mathrm{C}$, the reaction sections were incubated with the liquid mixture $(0.02 \%$ daminobenzidine tetrahydrochloride, $3 \% \mathrm{H}_{2} \mathrm{O}_{2}$, and $0.1 \% \mathrm{PBS}$ ). Finally, the sections were dehydrated and covered with coverslips. We examined the sections and counted the cells with strong or moderate brown staining, weak or no staining as positive or negative Foxj1 cells, respectively, from each group at higher magnified images. We took the average assays of each group as the valuable results.

\section{Quantitative Analysis}

Cells double labeled for Foxj1 and the other phenotypic markers used in the experiment were quantified. Sections were double labeled for Foxj1 and MAP-2 and GFAP. To identify the proportion of each phenotype-specific markerpositive cells expressing Foxj1, a minimum of 200 phenotypespecific marker-positive cells were counted adjacent to the wound in each section. Then double labeled cells for Foxj1 and phenotype-specific markers were recorded. Two or three adjacent sections per animal were sampled.

\section{Statistical Analysis}

All data were analyzed with Stata 7.0 statistical software. All values were expressed as mean \pm SEM. One-way ANOVA followed by the Tukey's post hoc multiple comparison tests was used for statistical analysis. $P$ values less than 0.05 were considered statistically significant. Each experiment consisted of at least three replicates per condition.

\section{Results}

The Expression of Foxj1 in Brain by Western Blot

In order to investigate the temporal patterns of Foxj1 expression after TBI, Western blot was performed in this study. In the cerebral cortex surrounding the injury, Foxj1 protein level was low in normal and sham cortex, and increased at $12 \mathrm{~h}$ after TBI and peaked at day 3, then gradually reduced thereafter; however, the expression of Foxj1 at day 28 after injury was still higher than the sham control. These data indicated that Foxj1 protein level had a temporally change after TBI (Fig. 1a and b).

The Changed Distribution of Foxj1 in the Brain Cortex after TBI

We used the coronal sections of the uninjured shamoperation and day 3 after injury to assess the changed distribution of the expression of Foxj1 by immunohistochemistry. We could see that the immunostaining of Foxj1 deposited strongly in the plasmalemma adjacent to the lesion site (Fig. 2e-g); however, a few positive immunostaining was found in the equal contralateral hemisphere (Fig. 2c, d, and g) and the uninjured sham-operated hemisphere (Fig. 2a and b). Further magnifications revealed clearly the distribution and the morphous of the positive immunostaining. The results suggested that the expression of Foxj1 was apparently higher in the ipsilateral injured brain on day 3 after TBI compared with the contralateral hemisphere and the sham-operated hemisphere.

The Colocalization of Foxj1 with Different Cellular

Markers by Double Immunofluorescent Staining

To further determine the cell types expressing Foxj1 after TBI, we used double labeling immunofluorescent staining with cell-specific markers: MAP-2 (a marker of neurons) and GFAP (a marker of astrocytes). We found that Foxj1 was expressed in neurons (Fig. 3a-c) and astrocytes (Fig. $3 \mathrm{~g}-\mathrm{i}$ ) and with a relatively low level in sham brain (Fig. $3 \mathrm{~d}-\mathrm{f}$ and $\mathrm{j}-\mathrm{l}$ ). To identify the proportion of each phenotype-specific marker-positive cells expressing Foxj1, a minimum of 200 phenotype-specific marker-positive cells were counted between sham and 3 days after TBI (Fig. $3 \mathrm{~m}$ ). After injury, Foxj1 expression was increased significantly in neurons and astrocytes at 3 days compared with sham brain.

Cellular Proliferation in the Brains after TBI

To identify the proliferative cell types after TBI, we performed double immunofluorescent staining with NeuN (a marker of neurons), GFAP (a marker of astrocytes), and Ki67 (a marker of cell division) in injured brain 3 days after TBI. We also performed Foxj1 and Ki67 to investigate their relationship. The results revealed that there were colocalizations between Neun and Ki67 (Fig. 4a-c), GFAP and Ki67 (Fig. 4d-f), Foxj1, and Ki67 (Fig. 4g-i), that was to 
Fig. 1 Western blot showing the temporal protein levels of Foxj1 following traumatic brain injury (TBI). Western blot was performed to assess the protein level of Foxj1 in the cortex surrounding the wound at different survival times after TBI. The bar chart below demonstrated the ratio of Foxj1 relative to GAPDH for each time point. a Foxj1 protein level was relatively lower in normal and sham cortex, and peaked at day 3 , then gradually reduced. b Semiquantitative analysis (relative optical density) showed the intensity of staining of Foxj1 to GAPDH for each time point. The data are means $\pm \operatorname{SEM}(n=3$, $* P<0.05$, significantly different from the sham-operated group)

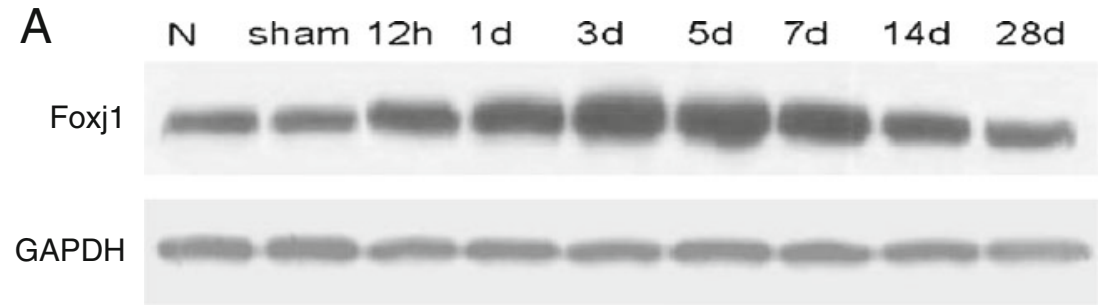

B

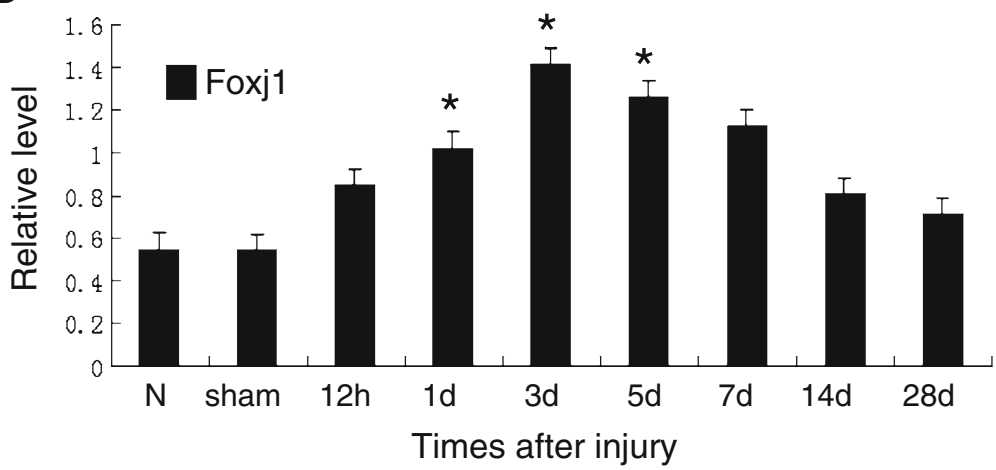

say neurons and astrocytes had proliferated after injury and Foxj1 had the relationship with cell proliferation.

\section{Discussion}

TBI has been one of the leading causes of death and disability in both industrialized and developing countries (Djebaili et al. 2004; Lo et al. 2003) and results in a significant society burden throughout the world (Langlois et al. 2006; Marshall 2000; Teasdale and Graham 1998). The pathophysiology of cerebral contusions is varied and complicated, including complex temporal and regional changes of cerebral blood flow and metabolism (Katayama et al. 1998; Bullock et al. 1992), disruption of the bloodbrain barrier resulting in brain edema (Unterberg et al. 2004), and progressive neuronal cell death in pericontusional tissue (Cervos-Navarro and Lafuente 1991). TBI induces deleterious neuroinflammation, as proved by edema, free radicals, cytokine production, induction of nitric-oxide synthase and cyclooxygenase type 2, and leukocyte infiltration (Xiao et al. 2008). Because a series of inner mechanisms leading to the physiological and the pathological alteration in the brain after injury have not been understood clearly, there are currently no good treatments that improve clinical outcome measures (Roberts et al. 1998; McKee et al. 2005). In our study, we employed a controlled stereotactic knife lesion model in adult rats to investigate the cell mechanism after TBI.

We displayed the increased expression of Foxj1 in adult rats' brain after traumatic brain injury for the first time.
Western blot analysis showed that the expression of Foxj1 was significantly increased and peaked at 3 days after injury. We also found that the staining of Foxj1 was enhanced obviously in the ipisilateral brain cortex nearby the lesion site compared to the conspilateral brain and the sham-operated brain by immuohistochemical staining. By double immunofluorescent staining, we observed that there were co-localization of Foxj1 and MAP-2, as well as Foxj1 and GFAP. In addition, the co-localization of Foxj1 with MAP-2 and GFAP was increased in the brain 3 days after injury compared to the sham brain. In our experiment, the important alteration of the expression of Foxj1 located in cytoplasm in the brain after injury forcefully supported the idea that Foxj1 was implicated in the central nervous system pathophysiology after TBI. Our findings may provide a crucial clue to learn the cellular and molecular mechanisms underlying TBI.

Traumatic brain injury would lead to permanent motor, cognitive and behavioral deficits, which are the result of neural tissue loss and cell death (Paghupathi et al. 2000). In response to the injury, the cells in the brain will produce corresponding reactions to resist the injury and help to recover the insulted functions. Neurons may be replenished by neural stem cells in the dentate gyrus and subventricular zones (Yagita et al. 2001; Peterson 2002). Astrocytes proliferate possibly to support surviving neurons and prevent further tissue damage through formation of the glial scar (Smith et al. 2001). Microglia increase to get rid of cellular debris and promote recovery of brain function (Glulian et al. 1991). Recent studies have shown that the mammalian brain contains neural stem and progenitor cells 
Fig. 2 The changed distribution of Foxj1 in injured brain cortex by immunohistochemical staining. a, b We could observe the staining of Foxj1 in the sham-operated brain, and the level was relatively lower. c, d The same staining was found in the equal contralateral hemisphere of the injured brain; however, the level was low as the sham brain. e, f We could see that the immunostaining of Foxj1 deposited strongly adjacent to the lesion site, in addition the level was significantly higher compared with the sham and the contralateral hemisphere of the brain after TBI. g Quantitative analysis of Foxj1 positive cells/ $\mathrm{mm}^{2}$ between contralateral and ipsilateral brains 3 days after injury. Foxj1 was significantly increased in the ipsilateral brain at 3 days after TBI. Asterisk indicated significant difference at $P<0.05$ compared with contralateral brain. Error bars SEM. Scale bars: left columns (a, c, e), $50 \mu \mathrm{m}$; right columns (b, d, f), $10 \mu \mathrm{m}$
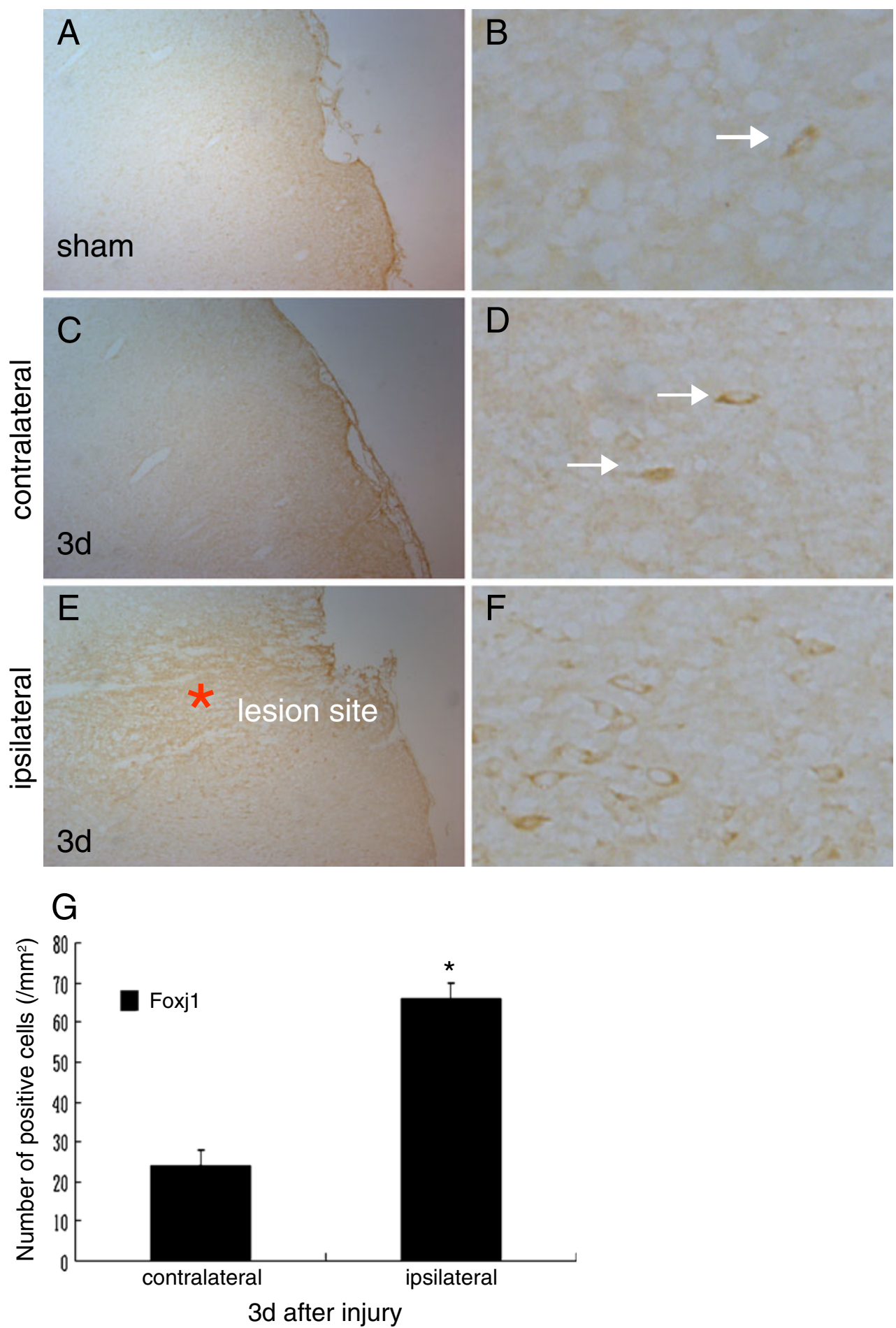

in different regions such as the sub-granular zone of the dentate gyrus and the SVZ of the lateral ventricles (Reynolds and Weiss 1992; Johansson et al. 1999; Alvarez-Buylla et al. 2002; Doetsch et al. 1999; Palmer et al. 1997). After injury, neurogenesis is stimulated in the adult brain (Kunlin et al. 2010) and migration toward pathology is the first critical step in stem cell engagement during regeneration (Imitola et al. 2004). Neural stem cells migrate through the parenchyma along nonstereotypical routes in a precise directed manner across great distances to injury sites in the central nervous system, where they could engage niches harboring local transiently expressed reparative signals (Imitola et al. 2004). Injury-induced neurogenesis has been observed across a broad range of injury models in experimental animals and human patients. For example, cerebral ischemia enhances proliferation of 

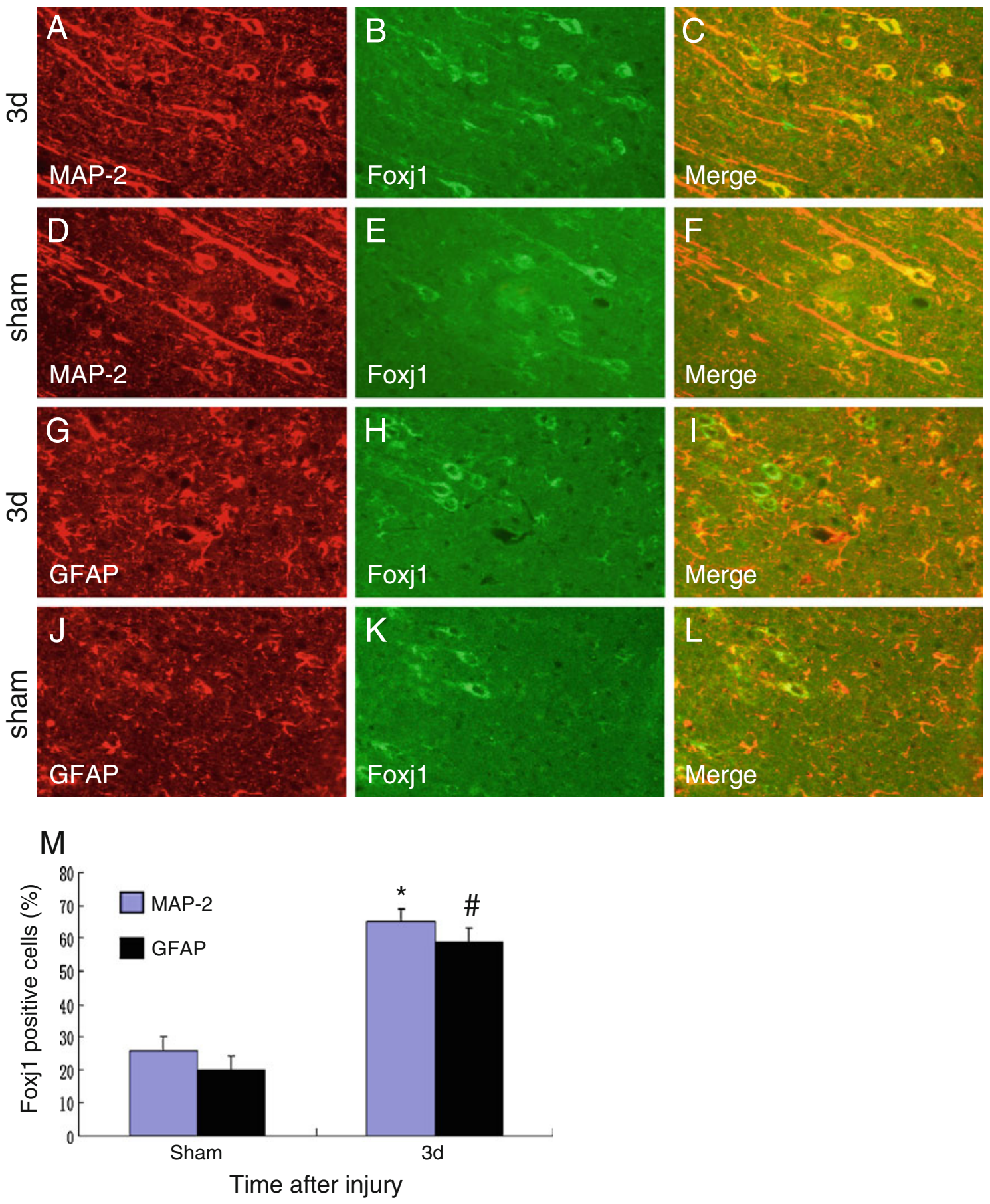

Fig. 3 Double immunofluorescence staining for Foxj1 and different phenotype-specific markers in adult rat brain 3 days after TBI. The sections from sham and injured brains 3 days after TBI were immunostained with Foxj1 (green, $\mathbf{b}, \mathbf{e}, \mathbf{h}, \mathbf{k}$ ) and different cell makers, such as MAP-2 (a marker of neurons, red, a, d) and GFAP (a marker of astrocytes, red, $\mathbf{g}, \mathbf{j}$ ), and the co-localization of Foxj1 with different phenotype-specific markers were visualized in the merged images $(\mathbf{c}, \mathbf{f}, \mathbf{i}, \mathbf{l})$. $\mathbf{a}-\mathbf{c}, \mathbf{g}-\mathbf{i}$ Immunostaining for Foxj1 with MAP-2 and
GFAP at 3 days after TBI; d-f, $\mathbf{j}-\mathbf{l}$ Immunostaining for Foxj1 with MAP-2 and GFAP in sham brain. $\mathbf{m}$ Quantitative analysis of different phenotype-specific markers positive cells expressing Foxj1 (\%) in sham group and 3 days after injury. The change of Foxj1 was striking in neurons and astrocytes; *, \# indicate significant difference at $P<$ 0.05 compared with the sham group. Error bars SEM. Scale bars $20 \mu \mathrm{m}(\mathbf{a}-\mathbf{I})$ 

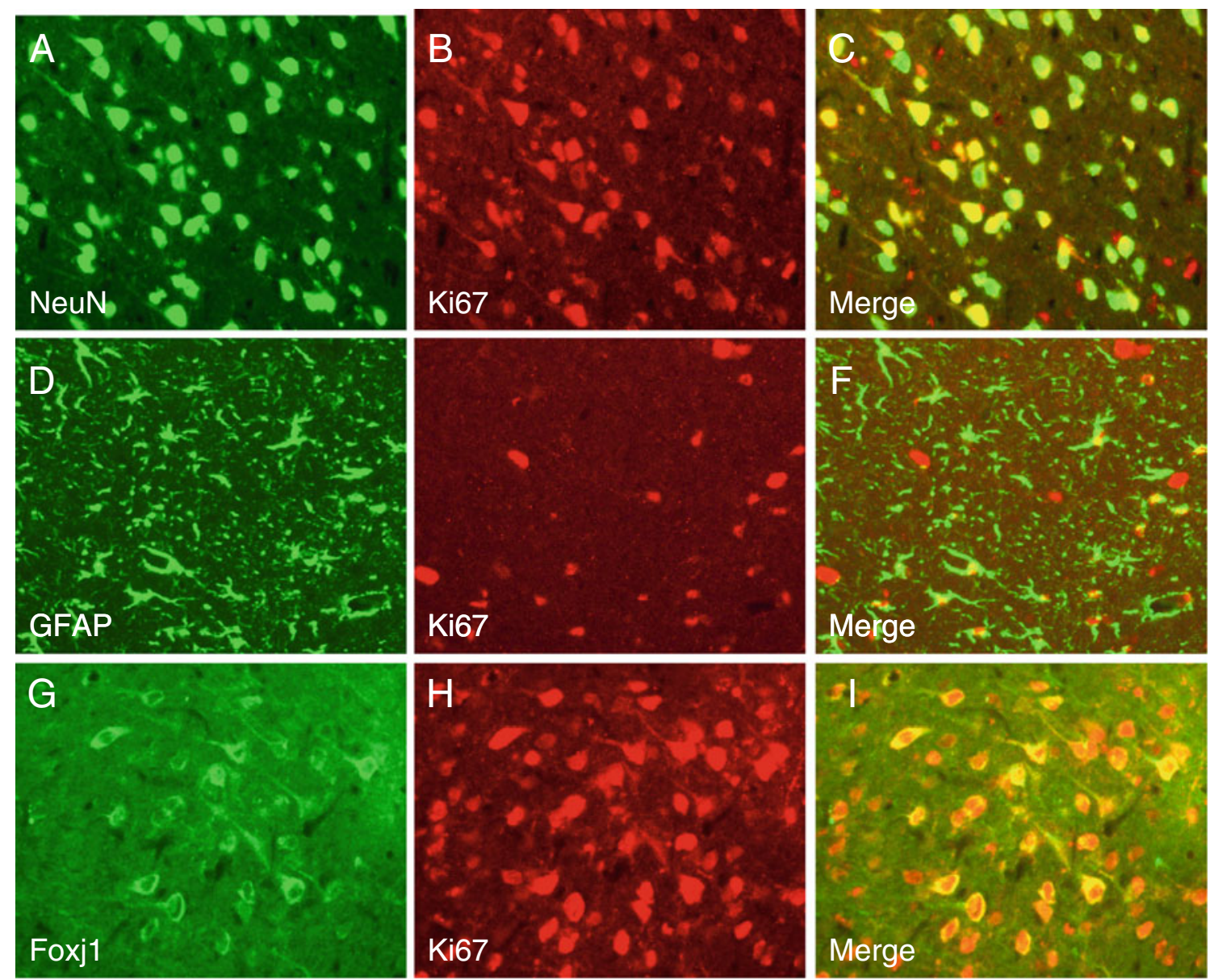

Fig. 4 Double immunofluorescence staining for cellular proliferation in the brains after TBI. Double immunofluorescence staining for NeuN (a marker of neurons, green, a), GFAP (green, d), Foxj1 (green, g), and Ki67 (a marker of cell division, red, b, e, h) in injured brain

neuronal precursor cells in the SVZ, followed by their migration into ischemic brain regions, where they differentiate and mature (Jin et al. 2001), in addition ectopic neurogenesis has been observed in animal models in the ipsilateral striatum of middle cerebral artery occlusion (Arvidsson et al. 2002; Zhang et al. 2002) and in degenerated hippocampal CA1 with global cerebral ischemia (Nakatomi et al. 2002; Bendel et al. 2005). The finding that brain injury stimulates the production of neurogenesis in the mammalian brain shows a role for this process in brain repair.

Foxj1 is a member of the Fox family of transcription factors, which plays an important role in cilia formation (Clevidence et al. 1993; Hackett et al. 1995; Brody et al. 2000), left-right axis determination of organs (Brody et al. 2000; Chen et al., 1998), suppressing $\mathrm{T}$ cell activity (Srivatsan and Peng 2005), inhibiting the humoral immune response in B cells (Lin et al. 2005) and so on. Recent studies found that Foxj1 is required for postnatal differentiation of ependymal cells and a subset of astrocytes in the cortex after TBI. In the rat brain 3 days after injury, there were colocalization between NeuN and Ki67 (a-c), GFAP and Ki67 (d-f), Foxj1 and Ki67 (g-i). Scale bars $20 \mu \mathrm{m}(\mathbf{a}-\mathbf{i})$

SVZ, where these cells form a postnatal neural stem cell niche (Jacquet and Salinas-Mondragon 2009). The findings of the studies revealed that the subset of astrocytes harvested from the SVZ generate neurospheres, which had the capability of self-renew and had the potential to give rise to neurons, astrocytes, and oligodendrocytes, thus functionally resembling adult neural stem cells. In support of this, recent studies have revealed that Foxj1 promoteractive cells in the spinal canal and SVZ participate in neurogenesis and gliogenesis in spinal cord injury and in response to stroke (Meletis et al. 2008; Carlen et al. 2009).

Our study revealed that the expression of Foxj1 was significantly increased in the rat brain after injury, which supported the concept that Foxj1 may be involved in the physiological and the pathological process in the injured brain. Recent studies have displayed that Foxj1 is depended in inducing the differentiation of a subset of astrocytes in the subventricular zone, which can functionally act as adult neural stem cells and give rise to neurons, astrocytes, and oligodendrocytes. Our results suggest that Foxj1 may be 
required for the differentiation of the cells acting as adult neural stem cell which participate in neurogenesis and give rise to neurons, astrocytes, and oligodendrocytes. These cells may migrate to the lesion region by stimulation of injury to compensate for the loss of neuronal function caused by TBI. Our experiment may provide a novel strategy for the treatment of CNS trauma in the field of neurogenesis. Further studies are needed to confirm the inherent mechanisms of the role of Foxj1 after brain injury.

Open Access This article is distributed under the terms of the Creative Commons Attribution Noncommercial License which permits any noncommercial use, distribution, and reproduction in any medium, provided the original author(s) and source are credited.

\section{References}

Alvarez-Buylla A, Seri B, Doetsch F (2002) Identification of neural stem cells in the adult vertebrate brain. Brain Res Bull 57:751-758

Arvidsson A, Collin T, Kirik D, Kokaia Z, Lindvall O (2002) Neuronal replacement from endogenous precursors in the adult brain after stroke. Nat Med 8:963-970

Bendel O, Bueters T, von Euler M, Ove Ogren S, Sandin J, von Euler G (2005) Reappearance of hippocampal CA1 neurons after ischemia is associated with recovery of learning and memory. $\mathrm{J}$ Cereb Blood Flow Metab 25:1586-1595

Brody SL, Yan XH, Wuerffel MK, Song SK, Shapiro SD (2000) Ciliogenesis and left-right axis defects in forkhead factor HFH-4null mice. Am J Respir Cell Mol Biol 23:45-51

Bullock R, Sakas D, Patterson J, Wyper D, Hadley D, Maxwell W, Teasdale GM (1992) Early post-traumatic cerebral blood flow mapping: correlation with structural damage after focal injury. Acta Neurochir Suppl (Wien) 55:14-17

Carlen M, Meletis K, Goritz C, Darsalia V, Evergren E, Tanigaki K, Amendola M, Barnabe-Heider F, Yeung MS, Naldini L (2009) Forebrain ependymal cells are Notch-dependent and generate neuroblasts and astrocytes after stroke. Nat Neurosci 12:259-267

Cervos-Navarro J, Lafuente JV (1991) Traumatic brain injuries: structural changes. J Neurol Sci 103(Suppl):S3-S14

Chen J, Knowles HJ, Hebert JL, Hackett BP (1998) Mutation of the mouse hepatocyte nuclear factor/forkhead homologue 4 gene results in an absence of cilia and random left-right asymmetry. J Clin Invest 102:1077-1082

Clevidence DE, Overdier DG, Tao W, Qian X, Pani L, Lai E, Costa RH (1993) Identification of nine tissue-specific transcription factors of the hepatocyte nuclear factor 3/forkhead DNA-binding domain family. Proc Natl Acad Sci USA 90:3948-3952

Di Giovanni S, Movsesyan V (2005) Cell cycle inhibition provides neuroprotection and reduces glial proliferation and scar formation after traumatic brain injury. Neuroscience 102:8333-8338

Djebaili M, Hoffman SW, Stein DG (2004) Allopregnanolone and progesterone decrease cell death and cognitive deficits after a contusion of the rat pre-frontal cortex. Neurosci 123:349-359

Doetsch F, Caille I, Lim DA, García-Verdugo JM, Alvarez-Buylla A (1999) Subventricular zone astrocytes are neural stem cells in the adult mammalian brain. Cell 97:703-716

Glulian D, Frederickson R, McGaugh J, Fehon D (1991) Microglianeuron interactions after injury to the central nervous system. Hogrefe and Huber, Lewiston, NY, pp 73-82
Hackett BP, Brody SL, Liang M (1995) Primary structure of hepatocyte nuclear factor/forkhead homologue 4 and characterization of gene expression in the developing respiratory and reproductive epithelium. Proc Natl Acad Sci 92:4249-4253

Imitola J, Raddassi K, Park Kook In, Mueller F-J (2004) Directed migration of neural stem cells to sites of CNS injury by the stromal cell-derived factor $1 \alpha / \mathrm{CXC}$ chemokine receptor 4 pathway. PNAS 101:18117-18122

Jacquet BV, Salinas-Mondragon R (2009) FoxJ1-dependent gene expression is required for differentiation of radial glia into ependymal cells and a subset of astrocytes in the postnatal brain. Development 136:4021-4031

Jin K, Minami M, Lan JQ, Mao XO, Batteur S, Simon RP, Greenberg DA (2001) Neurogenesis in dentate subgranular zone and rostral subventricular zone after focal cerebral ischemia in the rat. Proc Natl Acad Sci USA 98:4710-4715

Johansson CB, Momma S, Clarke DL, Risling M, Lendahl U, Frise'n $J$ (1999) Identification of a neural stem cell in the adult mammalian central nervous system. Cell 96:25-34

Katayama Y, Mori T, Maeda T, Kawamata T (1998) Pathogenesis of the mass effect of cerebral contusions: rapid increase in osmolality within the contusion necrosis. Acta Neurochir Suppl 71:289-292

Kunlin Jin, Xiaomei Wang, Lin Xie, Xiao Ou Mao, Greenberg DA (2010) Transgenic ablation of doublecortin-expressing cells suppresses adult neurogenesis and worsens stroke outcome in mice. PNAS 107:7993-7998

Langlois JA, Rutland-Brown W, Wald MM (2006) The epidemiology and impact of traumatic brain injury: a brief overview. J Head Trauma Rehabil 21:375-378

Lin L, Brody SL, Peng SL (2005) Restraint of B cell activation by Foxj1-mediated antagonism of NF-kB and IL-6. J Immunol 175:951-958

Lo EH, Dalkara T, Moskowitz MA (2003) Mechanisms, challenges and opportunities in stroke. Nat Rev Neurosci 4:399-415

Marshall LF (2000) Epidemiology and cost of central nervous system injury. Clin Neurosurg 46:105-112

McGraw J, Hiebert GW, Steeves JD (2001) Modulating astrogliosis after neurotrauma. J Neurosci Res 63:109-115

McKee JA, Brewer RP, Macy GE, Borel CO, Reynolds JD, Warner DS (2005) Magnesium neuroprotection is limited in humans with acute brain injury. Neurocrit Care 2:342-351

Meletis K, Barnabe-Heider F, Carlen M, Evergren E, Tomilin N, Shupliakov O, Frisen J (2008) Spinal cord injury reveals multilineage differentiation of ependymal cells. PLoS Biol 6: e182

Nakatomi H, Kuriu T, Okabe S, Yamamoto S, Hatano O, Kawahara N, Tamura A, Kirino T, Nakafuku M (2002) Regeneration of hippocampal pyramidal neurons after ischemic brain injury by recruitment of endogenous neural progenitors. Cell 110:429441

Nortje J, Menon DK (2004) Traumatic brain injury: physiology, mechanisms, and outcome. Curr Opin Neurol 17:711-718

Paghupathi R, Graham D, Mcintosh TK (2000) Apoptosis after traumatic brain injury. J Neurontrauma 17:927-938

Palmer TD, Takahashi J, Gage FH (1997) The adult rat hippocampus contains primordial neural stem cells. Mol Cell Neurosci 8:389404

Peterson DA (2002) Stem cells in brain plasticity and repair. Curr Opin Pharmacol 2:34-42

Plesnila N, von Baumgarten L, Retiounskaia M (2007) Delayed neuronal death after brain trauma involves p53-dependent inhibition of NF- $\kappa \mathrm{B}$ transcriptional activity. Cell Death Differ 14:1529-1541

Raghupathi R (2004) Cell death mechanisms following traumatic brain injury. Brain Pathol 14:215-222 
Reynolds BA, Weiss S (1992) Generation of neurons and astrocytes from isolated cells of the adult mammalian central nervous system. Science 255:1707-1710

Roberts I, Schierhout G, Alderson P (1998) Absence of evidence for the effectiveness of five interventions routinely used in the intensive care management of severe head injury: a systematic review. J Neurol Neurosurg Psychiatry 65:729-733

Smith C, Berry M, Clarke WE, Logan A (2001) Differential expression of fibroblast growth factor-2 and fibroblast growth factor receptor 1 in a scaning and nonscaning model of CNS injury in the rat. J Neurosci 13:443-456

Srivatsan S, Peng SL (2005) Cutting edge: Foxj1 protects against autoimmunity and inhibits thymocyte egress. J Immunol 175:7805-7809

Teasdale GM, Graham DI (1998) Craniocerebral trauma: protection and retrieval of the neuronal population after injury. Neurosurgery 43:723-738

Unterberg AW, Stover J, Kress B, Kiening KL (2004) Edema and brain trauma. Neuroscience 129:1021-1029
Walker PA, Shah SK, Harting MT (2009) Progenitor cell therapies for traumatic brain injury: barriers and opportunities in translation. Disease Models \& Mechanisms 2:23-38

Xiao Ru, Chen VC, Besson TB (2008) Combination therapy with fenofibrate, a peroxisome proliferator-activated receptor $\alpha$ agonist, and simvastatin, a 3-hydroxy-3-methylglutaryl-coenzyme a reductase inhibitor, on experimental traumatic brain injury. J Pharmacol Exp Ther 326:966-974

Yagita Y, Kitagawa K, Ohtsuki T (2001) Neurongenesis by rogenitor cells in the ischemic adult rat hippocampus. Stroke 2001 (32):1890-1896

Zhang R, Wang Y, Zhang L, Zhang Z, Tsang W, Lu M, Zhang L, Chopp M (2002) Sildenafil (Viagra) induces neurogenesis and promotes functional recovery after stroke in rats. Stroke 33:2675-2680

Zweckberger K, Eros C, Zimmermann R, Kim SW, Engel D, Plesnila N (2006) Effect of early and delayed decompressive craniectomy on secondary brain damage after controlled cortical impact in mice. J Neurotrauma 23:1083-1093 